\title{
UV ENERGY DISTRIBUTIONS OF ACTIVE COOL STARS.
}

\author{
C. MOROSSI AND M. FRANCHINI
}

Osservatorio Astronomico di Trieste, Via Tiepolo 11, I-34131, Trieste, Italy

AND

\author{
M.L. MALAGNINI \\ Dipartimento di Astronomia, Università degli Studi di Trieste, \\ Via Tiepolo 11, I-34131, Trieste, Italy
}

\begin{abstract}
We present semi-empirical models based on the "minimumtemperature-concept" in order to provide a description of the UV spectra of solar type stars consistent with the observational data.
\end{abstract}

\section{Introduction}

The analysis of spectral energy distributions (SED's) of cool stars received a strong incentive in the last years from the availability of improved theoretical flux distributions (Kurucz 1993, hereafter K93; Edvardsson et al. 1993, hereafter EAGLNT). Recently, the predicted theoretical fluxes have been compared with observations of K (Malagnini et al. 1992, Morossi et al. 1993), F, and G stars (EAGLNT). The main result of the analysis of the K stars is the failure of K93 models in predicting the near UV $(2200<\lambda<3000 \AA)$ SED's of stars showing chromospheric features. Morossi et al. (1993) presented semi-empirical models (hereafter Tmin models), based on a modified temperature structure with respect to the K93 one, obtained by taking into account the presence of a minimum temperature region $\left(T=T_{\min }\right)$ in the upper photosphere. The fluxes computed starting from this new set of models provide a fairly good description of the observations not only in the visible and IR regions but also in the UV. In this paper we will extend the "minimum-temperature-concept" approach to effective temperatures higher than those studied in Morossi et al. (1993). In 

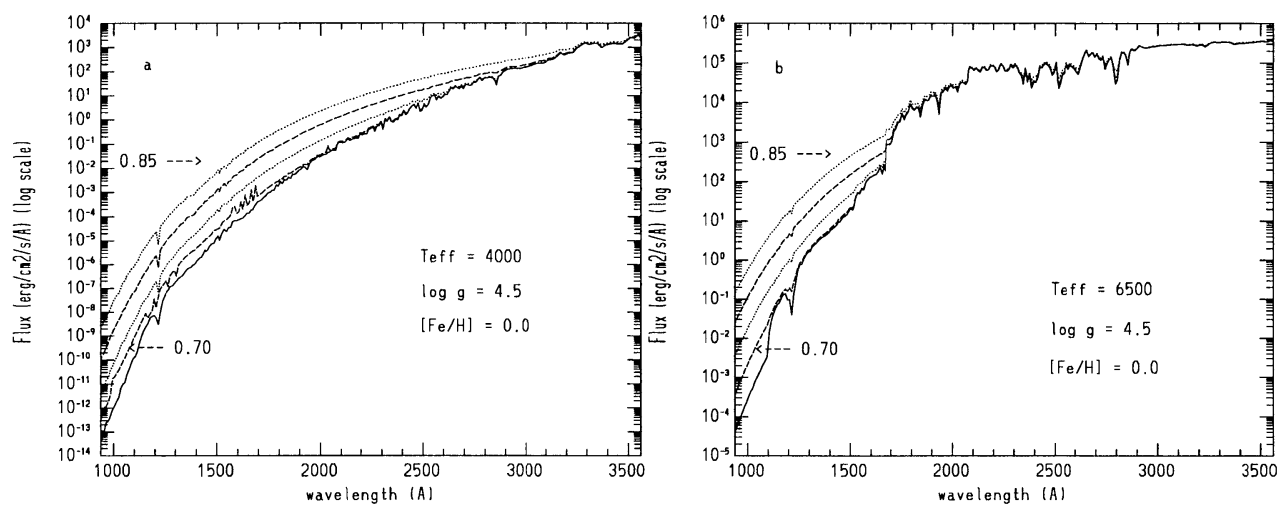

Figure 1. Ultraviolet fluxes computed from K93 "standard" models (solid lines), and from Tmin models with four different values of $R=\frac{T_{\min }}{T_{\text {eff }}}$ (i.e. $0.70,0.75,0.80,0.85$ ). The cases $T_{\text {eff }}=4000 \mathrm{~K}$ and $T_{\text {eff }}=6500 \mathrm{~K}(\log g=4.5 \mathrm{dex})$ are shown in a) and b) respectively

particular, we will analyse a set of stars which are proposed as "candidate solar analogs" (see Cayrel de Strobel 1996 for further details).

\section{Tmin models}

The nine K93 models characterized by the $T_{\text {eff }}, \log g$ and $[M / H]$ values given in Table 1 have been modified according to the following formula:

$$
T_{\mathrm{emp}}(\tau)=\max \left(T(\tau), R \times T_{\mathrm{eff}}\right)
$$

where $\mathrm{R}$ is the ratio $\frac{T_{\min }}{T_{\text {eff }}}$. The ATLAS9 code was then used to compute the energy distributions. Figure 1 shows the UV SED's for two $T_{\text {eff's for }}$ illustrating the differences introduced by the presence of a Tmin region in the $T(\tau)$ atmosphere structure. It results that the use of Tmin models produces an enhancement of the ultraviolet fluxes due to a lower blanketing. The higher the $\frac{T_{\min }}{T_{\text {eff }}}$ ratios, the larger the effect. The wavelength shorter of which the increase in the UV fluxes becomes significant depends mainly on the $T_{\text {eff }}$ of the original K93 model. The higher the $T_{\text {eff }}$, the shorter the limiting wavelength.

\section{Observations versus predictions}

In this paper we pay attention to a narrow temperature range around the solar value. EAGLNT introduced six UV bands (see their Table 5): at the shortest wavelength, band (A), they found that "the model flux is typically 
TABLE 1. Program stars and results

\begin{tabular}{rrrrrl}
\hline $\mathrm{HD}$ & $\mathrm{B}-\mathrm{V}$ & $T_{\text {eff }}$ & $\log g$ & {$[\mathrm{Fe} / \mathrm{H}]$} & $\frac{T_{\min }}{T_{\text {eff }}}$ \\
\hline 1835 & 0.66 & 5781. & 4.50 & 0.18 & 0.83 \\
10307 & 0.62 & 5538. & 4.40 & 0.07 & 0.85 \\
20630 & 0.68 & 5631. & 4.45 & 0.02 & 0.85 \\
25680 & 0.62 & 5794. & 4.30 & -0.03 & 0.84 \\
30495 & 0.63 & 5915. & 4.40 & -0.02 & 0.82 \\
44594 & 0.66 & 5777. & 4.40 & 0.15 & 0.80 \\
72905 & 0.62 & 5843. & 4.40 & -0.11 & 0.85 \\
95128 & 0.61 & 5860. & 4.31 & -0.02 & 0.81 \\
186427 & 0.66 & 5765. & 4.30 & 0.05 & 0.80 \\
\hline
\end{tabular}

TABLE 2. Comparison of Tmin and K93 model predictions

\begin{tabular}{rrrrrrrr}
\hline$T_{\text {eff }}$ & $\frac{T_{\min }}{T_{\text {eff }}}$ & $\mathrm{A}$ & $\mathrm{B}$ & $\mathrm{C}$ & $\mathrm{D}$ & $\mathrm{E}$ & $\mathrm{F}$ \\
\hline 6500 & 0.85 & -0.76 & -0.22 & -0.11 & -0.06 & -0.04 & -0.02 \\
& 0.80 & -0.37 & -0.06 & -0.02 & -0.02 & -0.01 & -0.00 \\
& 0.75 & -0.10 & -0.00 & -0.00 & -0.00 & -0.00 & -0.00 \\
& 0.70 & -0.00 & 0.00 & 0.00 & 0.00 & 0.00 & 0.00 \\
6000 & 0.85 & -1.34 & -0.72 & -0.43 & -0.12 & -0.06 & -0.04 \\
& 0.80 & -0.88 & -0.35 & -0.16 & -0.04 & -0.02 & -0.01 \\
& 0.75 & -0.42 & -0.11 & -0.04 & -0.00 & -0.00 & -0.00 \\
& 0.70 & -0.14 & -0.03 & -0.01 & -0.00 & -0.00 & -0.00 \\
5500 & 0.85 & -1.75 & -1.12 & -0.79 & -0.26 & -0.08 & -0.06 \\
& 0.80 & -1.23 & -0.65 & -0.38 & -0.08 & -0.02 & -0.01 \\
& 0.75 & -0.67 & -0.24 & -0.10 & -0.01 & -0.00 & 0.00 \\
& 0.70 & -0.18 & -0.03 & -0.00 & 0.00 & 0.00 & 0.00 \\
\hline
\end{tabular}

five times lower than the observed fluxes". Analogously, we computed in the same bands the logarithms of the ratios between K93 and Tmin model fluxes for $T_{\text {eff }}=5750 \mathrm{~K}$ (see Table 2).

The ratios in Table 2 show the same trend with wavelength as those reported in EAGLNT Table 5. Actually, a good agreement between observations and theoretical fluxes can be obtained by using Tmin models characterized by the $R$ values given in Table 1 (see for example fig. 2).

Our analyses suggest that, for active cool stars, Tmin models are well suited for interpreting the observed ultraviolet fluxes. Our results may also explain why, for the Sun, EAGLNT do not find deviations in the A passband between observations and their solar model. In fact, assuming that the Tmin 



Figure 2. Observed and predicted fluxes for the star HD 25680 (see Table 1 and text)

models are required to take into account stellar activity, the agreement found by EAGLNT in the case of the sun can be understood since the far UV solar observations they used "correspond to regions of the quiet Sun, where the inhomogeneities have been smoothed out and all active regions avoided" (Samain 1979).

\section{Acknowledgements}

Partial support from CRA 60\% (Osservatorio Astronomico di Trieste) and from MURST $40 \%$ and $60 \%$ (Università degli Studi di Trieste) grants is acknowledged.

\section{References}

Cayrel de Strobel, G. 1996, A\&AR 7,243

Edvardsson B., Andersen J., Gustafsson B., Lambert D.L., Nissen P.E., Tomkin J. 1993, A\&A 275, 101 (EAGLNT)

Kurucz R.L. 1993, CD-ROM 13, ATLAS9 Stellar Atmosphere Programs and 2 km/s Grid (Cambridge: Smithsonian Astrophys. Obs.) (K93)

Malagnini M.L., Morossi C., Buser R., Parthasarathy M. 1992, A\&A 261, 558

Morossi C., Franchini M., Malagnini M.L., Kurucz R.L., Buser R. 1993, A\&A 277, 173

Samain D. 1979, A\&A 74, 225

\section{DISCUSSION}

ANDREAS KELZ: Do you expect that the 'minimum-temperature concept' would be a good description for main sequence stars hotter than $K$ and $G$ as well? Alternatively, for which stars wouldn't the Tmin layer make any difference in the UV?

CARLO MOROSSI: I still have to look at F type stars, but I suppose that for these kind of stars you'll have to check EUV fluxes, probably shortward of $912 \AA$, which is very difficult from an observational point of view. 